\title{
Harmine promotes periodontal ligament cell-induced tissue Regeneration
}

\author{
Lim, Hyun-Chang ; Cha, B-Y ; Song, S U ; Yun, J-H
}

\begin{abstract}
OBJECTIVE to investigate whether harmine has a promotive effect on human periodontal ligament cells (hPDLCs)-induced tissue regeneration. MATERIALS AND METHODS Various concentrations of harmine on hPDLCs proliferation were tested. Osteogenic and cementogenic characteristics were examined in hPDLC/rhBMP2 and hPDLC/harmine by alizarin red S staining, real-time PCR, and Western blotting assay. The activity of harmine was investigated in an ectopic transplantation nude mouse model. RESULTS We determined that 10 $\mu \mathrm{M}$ of harmine was the threshold concentration. hPDLC/harmine showed similar mineralized nodule formation in alizarin S staining compared to hPDLC/rhBMP-2. In real-time PCR, the highest gene expression level was observed for Runx2 in hPDLC/harmine at all time points. The level of CEMP-1 in hPDLC/harmine was higher at 7 days than hPDLCs alone. Thicker band of Runx2 in hPDLC/harmine was observed than in hPDLC/rhBMP-2 at 7 days by Western blotting. The band for CEMP-1 in hPDLC/harmine was thicker than hPDLCs alone at both 7 and 14 days. In ectopic transplantation, hPDLCs with harmine showed a comparable amount of mineralized tissue formation compared to rhBMP-2. hPDLCs with harmine or rhBMP-2 formed both bone and cementumlike tissue with Sharpey's fiber-like collagen insertion. CONCLUSION Harmine can be a potential candidate for promoting hPDLCs-induced tissue regeneration.
\end{abstract}

DOI: https://doi.org/10.1111/odi.12770

Posted at the Zurich Open Repository and Archive, University of Zurich ZORA URL: https://doi.org/10.5167/uzh-158029

Journal Article

Accepted Version

Originally published at:

Lim, Hyun-Chang; Cha, B-Y; Song, S U; Yun, J-H (2018). Harmine promotes periodontal ligament cell-induced tissue Regeneration. Oral Diseases, 24(3):456-464.

DOI: https://doi.org/10.1111/odi.12770 


\section{Harmine promotes periodontal ligament cell-induced tissue regeneration}

Hyun-Chang Lim ${ }^{1,2}$, Byung-Yoon $\mathrm{Cha}^{3}$, Sun U. Song ${ }^{4}$, Jeong-Ho Yun ${ }^{5,6}$

${ }^{1}$ Department of Periodontology, School of Dentistry, Kyung Hee University, Seoul, Republic of Korea

${ }^{2}$ Clinic for Fixed and Removable Prosthodontics and Dental Material Science, University of Zürich, Zürich, Switzerland

${ }^{3}$ Research Institute for Biological Functions, CHUBU University, Aichi, Japan

${ }^{4}$ Clinical Research Center, School of Medicine, Inha University, Incheon, Republic of Korea

${ }^{5}$ Department of Periodontology, School of Dentistry and Institute of Oral Bioscience, Chonbuk National University, Jeonju, Republic of Korea.

${ }^{6}$ Research Institute of Clinical Medicine of Chonbuk National University-Biomedical Research Institute of Chonbuk National University Hospital, Jeonju, Republic of Korea

Running title: Natural product for periodontal regeneration

Keywords: Harmine, Stem cell, Regenerative medicine, Periodontium, Periodontal ligament

\section{Corresponding author:}

Jeong-Ho Yun

Department of Periodontology, School of Dentistry, Chonbuk National University, 567 Baekje-daero, Deokjin-gu, Jeonju-si, Jeollabuk-do, 54896 Republic of Korea

Tel.: +82-63-2502289

Fax: +82-63-2502289

E-mail:grayheron@hanmail.net 
Date of submission (major revision): July 29, 2017 


\section{Abstract}

Objective: to investigate whether harmine has a promotive effect on human periodontal ligament cells (hPDLCs)-induced tissue regeneration.

Materials and Methods: Various concentrations of harmine on hPDLCs proliferation were tested. Osteogenic and cementogenic characteristics were examined in hPDLC/rhBMP-2 and hPDLC/harmine by alizarin red S staining, real-time PCR, and Western blotting assay. The activity of harmine was investigated in an ectopic transplantation nude mouse model.

Results: We determined that $10 \mu \mathrm{M}$ of harmine was the threshold concentration. hPDLC/harmine showed similar mineralized nodule formation in alizarin $\mathrm{S}$ staining compared to hPDLC/rhBMP-2. In real-time PCR, a highest gene expression level was observed for Runx2 in hPDLC/harmine at all time points. The level of CEMP-1 in hPDLC/harmine was higher at 7days than hPDLCs alone. Thicker band of Runx2 in hPDLC/harmine was observed than in hPDLC/rhBMP-2 at 7 days by Western blotting. The band for CEMP-1 in hPDLC/harmine was thicker than hPDLCs alone at both 7 and 14 days. In ectopic transplantation, hPDLCs with harmine showed a comparable amount of mineralized tissue formation compared to rhBMP-2. hPDLCs with harmine or rhBMP-2 formed both bone and cementum-like tissue with Sharpey`s fiber-like collagen insertion.

Conclusion: Harmine can be a potential candidate for promoting hPDLCs-induced tissue regeneration. 


\section{Introduction}

Periodontitis, one of the most widespread diseases worldwide, involves loss of toothsupporting structures in varying degrees and may cause loss of tooth, which deteriorates the quality of life (Cunha-Cruz et al., 2007). Considering the age-related prevalence of periodontitis, managing this disease has become increasingly important in the current aged and ultra-aged populations (Kassebaum et al., 2014).

Non-surgical, surgical and antimicrobial approaches or a combination of these approaches are conventional modes for resolution of periodontitis in a routine clinical setting (Dentino et al., 2013). Disappointingly, while these methods are effective for controlling periodontal disease, they are not sufficient for predictably achieving regeneration (Zeichner-David, 2006). Thus, various bioactive agents have been extensively explored. Growth factors, such as fibroblast growth factor-2, platelet-derived growth factor-BB and growth/differentiation factor-5 have been shown to have favorable preclinical and clinical results (Cochran et al., 2016, Lee \& Wikesjo, 2014, Nevins et al., 2005, Takayama et al., 2001), but they are still not a commonly used modality. Enamel matrix derivative has also been extensively investigated (Miron et al., 2016). Numerous studies have supported the regenerative outcome of enamel matrix derivative, but its effect has only been validated in limited environments (Miron et al., 2016). Moreover, folk medicine using natural compounds has driven the interest in periodontal regeneration; however, the number of studies is relatively insufficient.

$\beta$-carboline alkaloids, such as harmine, harmol, harmane, harmaline, harmalol, are of interest due to a large variety of pharmacologic effects (Allen \& Holmstedt, 1980, Cao et al., 2007). They are found in several plants, such as Apocynaceae, Elaeagnaceae, Leguminosae, Passifloraceae and Zygophyllaceae, and are empirically used as medicinal substances from 
the past: emmenagogue and abortifacient in the Middle East and North Africa, hallucinogenic in the Amazon, and a cure for gastrointestinal tract cancer and malaria in northwest China (Cao et al., 2007). In line with the above usage, many pharmacologic properties have been identified, such as monoamine oxidase inhibitory, vasorelaxant, antioxidation, and antimutagenic effects (Berrougui et al., 2006, Kim et al., 1997, Moura et al., 2007). Recently, the inhibition of osteoclast differentiation and the promotion of osteoblast differentiation have been serially demonstrated as effect of harmine, one of the $\beta$-carboline alkaloids (Yonezawa et al., 2011a, Yonezawa et al., 2011b). Interestingly, the bone anabolic effect of harmine may be derived from a structural difference with other $\beta$-carboline alkaloids and such an effect is not correlated with other pharmacologic actions. In this context, harmine can be a promising cue for periodontal tissue regeneration.

To the best of our knowledge, there has been little evidence to date that harmine could be used for the regeneration of tooth-supporting structures. The aim of the present study was to investigate whether harmine has a promotive effect on human periodontal ligament cells (hPDLCs)-induced tissue regeneration in vitro and in vivo.

\section{Materials and Methods}

\section{Periodontal ligament cells (PDLCs) isolation}

The study protocol for PDL cell isolation was approved by the Ethics Committee of Inha Hospital (Approval No. IUH IRB 11-32). Informed consent was obtained from a healthy candidate scheduled for third molar extraction. The isolation process for human PDLCs (hPDLCs) was described in a previous study (Jung et al., 2013, Kim et al., 2016, Prince et al., 
2001). PDLCs were obtained from the third molar extracted from systemically healthy donor with no history of smoking. The extracted third molar was washed using $\boldsymbol{\alpha}$-minimum essential medium (Gibco, Grand Island, NY) containing $100 \mathrm{U} / \mathrm{mL}$ penicillin and $100 \mathrm{mg} / \mathrm{mL}$ streptomycin. The middle 1/3- apical 1/3 of root surface was scraped to obtain PDL tissues. Then, the tissue was minced, and digested with $2 \mathrm{mg} / \mathrm{mL}$ collagenase (Waco Pure Chemical Industries, Tokyo, Japan) and $1 \mathrm{mg} / \mathrm{mL}$ dispase (Gibco) four times at an interval of 20 minutes at $37^{\circ} \mathrm{C}$. The collected cells were seeded into a T75 cell culture flask (BD Falcon Labware, Franklin Lakes, NJ) and cultured in a growth medium comprising a-minimum essential medium (Gibco), 15\% fetal bovine serum (Gibco), 2mM L-glutamine (Gibco), 100 $\mu \mathrm{M}$ ascorbic-acid-2-phosphate (Sigma-Aldrich, St. Louis, MO), $100 \mathrm{U} / \mathrm{mL}$ penicillin, and $100 \mathrm{mg} / \mathrm{mL}$ streptomycin (Gibco) at $37^{\circ} \mathrm{C}$ in a humidified atmosphere of $5 \% \mathrm{CO}_{2}$. The media was refreshed every 3 to 4 days. Cells at passages five were used in following experiments for the present study. The stemness of the cells used in the present study was already verified in our previous studies using same cells (Jung et al., 2013, Jung et al., 2014). In those studies, similar expression of stem cell surface markers, such as CD44, CD105, CD146, STRO-1 were detected by flow cytometry.

\section{Cell proliferation at various concentrations of harmine (MTT assay)}

hPDLCs were plated at $8.0 \times 10^{3}$ into 24 -well plates, and cultured in a growth medium containing different concentrations of harmine $(0,1,2.5,5,10,20$ and $40 \mu \mathrm{M})$ (Wako) for 3 , 7 and 14 days, respectively. Cellular activity was evaluated using 3-(4,5-dimethylthiazol-2yl)-2,5-diphenyl-2H-tetrazolium bromide (MTT) solution $(0.5 \mathrm{mg} / \mathrm{mL}$ ) (Amresco, Solon, $\mathrm{OH})$. Following a 3-hour incubation, the supernatant was removed and cells were treated with dimethyl sulfoxide (Amresco). Optical densities were measured at $540 \mathrm{~nm}$ using a microplate reader (Molecular Devices, Sunnyvale, CA). 


\section{Osteogenic and cementogenic induction of hPDLC/BMP-2 and hPDLC/harmine}

\section{Mineralized nodule formation}

hPDLCs $\left(5 \times 10^{4}\right.$ cells/well $)$ were cultured in 24 -well plates with an osteogenic differentiation medium containing either $100 \mathrm{ng} / \mathrm{mL}$ of rhBMP-2 (Novosis, Daewoong, Seoul, Korea) (hPDLSC/BMP) or $10 \mathrm{uM}$ of harmine (hPDLSC/Har) for 14 and 28 days. The osteogenic differentiation medium comprised a growth medium containing $10^{-8} \mathrm{M}$ dexamethasone (Sigma-Aldrich) and $50 \mu \mathrm{g} / \mathrm{ml}$ ascorbic acid (Gibco) and $10 \mathrm{mM} \beta$ glycerophosphate (Gibco), and was refreshed every 3 days. After the cellular matrices were rinsed with PBS, they were fixed with 4\% paraformaldehyde for 10 minutes, and then stained with $2 \%$ alizarin red S (pH 7.2) (Sigma-Aldrich) for 1 hour. Stained nodules were observed with a light microscope (Olympus CK41; Olympus Optical, Tokyo, Japan). The stained nodules were washed with PBS and treated with $10 \%$ cetylpyridinium chloride $(1 \mathrm{~mL} /$ well $)$ overnight to extract the staining agent. Then, the optical density of each group was measured at $540 \mathrm{~nm}$ using a microplate reader.

\section{Real-time polymerase chain reaction (PCR) for osteogenic and cementogenic markers}

hPDLCs $\left(2 \times 10^{5}\right.$ cells/well $)$ were cultured in 6-well plates with a growth medium containing $50 \mu \mathrm{g} / \mathrm{ml}$ ascorbic acid (Gibco) and $10 \mathrm{mM} \beta$-glycerophosphate (Gibco). Either $100 \mathrm{ng} / \mathrm{mL}$ of rhBMP-2 or $10 \mathrm{uM}$ of harmine was also added in the medium. The cell culture was performed for 1, 3, 7 and 10 days. The total RNA was isolated using the TRIzol reagent (Invitrogen, Carlsberg, CA) and cDNA was synthesized with an oligo (dT) primer (Maxime RT Premix; iNtRon Biotechnology, Daejeon, Korea). Real-time PCR was performed with the SYBR Green Master Mix (Applied Biosystems, Carlsbad, CA) in the StepOnePlus real-time PCR system (Applied Biosystems) in triplicate. The PCR amplification was conducted using 
the following target gene markers (Supplement 1): alkaline phosphatase $(A L P)$, osteocalcin $(O C N)$, Runt-related transcription factor 2 (Runx2) and cementum protein 1 (CEMP1). The relative mRNA expression of the above genes was calculated by comparison to the reference $(G A P D H)$.

\section{Western blotting}

Western blotting was performed for CEMP1, ALP, Runx2 and OCN. hPDLCs $\left(4 \times 10^{5}\right.$ cells/well) were cultured in a $60-\mathrm{mm}$ dish with a growth medium containing $50 \mu \mathrm{g} / \mathrm{ml}$ ascorbic acid (Gibco) and $10 \mathrm{mM} \beta$-glycerophosphate (Gibco) for 7 and 14 days. In the medium, either $100 \mathrm{ng} / \mathrm{mL}$ of rhBMP-2 or $10 \mathrm{uM}$ of harmine was supplemented. M-PER mammalian protein extraction reagent (Thermo Scitific, Odessa, TX, USA) was used for the extraction of proteins. The protein concentration of the lysates was determined using the Pierce BCA protein assay kit (Thermo Scitific). Following boiling for $10 \mathrm{~min}, 30 \mu \mathrm{g}$ of protein from each day was loaded on $10 \%$ sodium dodecyl sulfate (SDS) polyacrylamide gel electrophoresis (PAGE) with molecular weight marker, and separated by electrophoresis. Then the proteins were transferred to polyvinylidene difluoride (PVDF) membranes, followed by blocking for $60 \mathrm{~min}$ at room temperature in $0.1 \%$ Tween 20 -Tris-buffered saline containing 5\% skim milk, and incubating overnight at $4^{\circ} \mathrm{C}$ with anti-OCN (diluted 1:1,000, Santa Cruz Biosciences, Texas, USA), anti-Runx2 (diluted 1:2000, Abcam Ltd), antiCEMP1(diluted 1:1000, Santa Cruz Biosciences), anti-ALP (diluted 1:1000, Santa Cruz Biosciences), and anti- $\beta$-actin (Santa Cruz Biosciences) antibodies. The membranes were washed with $0.1 \%$ Tween 20-Tris-buffered saline three times for $5 \mathrm{~min}$, and then the membranes were incubated with donkey anti-goat horseradish peroxidase (HRP)-conjugated antibody for CEMP1 (diluted 1:10,000, Santa Cruz Biosciences), goat anti-rabbit HRPconjugated antibody for ALP, OCN and Runx2 (diluted 1,10,000, Santa Cruz Biosciences), 
goat anti-mouse HRP-conjugated antibody for $\beta$-actin (diluted 1:10,000, Santa Cruz Biosciences) at room temperature for 1 hour. The chemiluminescent reagent (Amersham Life Science) was applied and exposed to X-ray film (AGFA, Belgium).

\section{Subcutaneous ectopic transplantation}

All procedures for animal experiments were approved by the Institutional Animal Care and Use Committee of Inha University (Approval No.: INHA 140801-322). Twenty immunocompromised mice (6-week-old Balb/c nude mice, OrientBio, Sungnam, Korea) were used for ectopic transplantation. Eighty milligrams of biphasic calcium phosphate (BCP; MBCP Plus, Biomatlante, Bretagne, France) was used for the cell carrier. In all animals, three dorsal sites were selected and assigned as follows ( $\mathrm{n}=5$ per group):

Group1. BCP carrier only (BCP).

Group 2. BCP carrier-loaded rhBMP-2 (BCP/BMP).

Group 3. hPDLCs seeded on a BCP carrier (BCP/hPDLC).

Group 4. hPDLCs seeded on a rhBMP-2-loaded BCP carrier (BCP/hPDLC/BMP).

Group 5. BCP carrier-loaded harmine (BCP/Har).

Group 6. hPDLCs seeded harmine-loaded BCP carrier (BCP/hPDLC/Har).

The BCP carrier was incubated in PBS only, $1 \mu \mathrm{g}$ of rhBMP-2 in PBS (Jung et al., 2014), and $16.98 \mu \mathrm{g}$ of harmine in PBS overnight at $37^{\circ} \mathrm{C}$. The loading amount of harmine was determined by the threshold concentration from MTT assay $(10 \mu \mathrm{M})$ and the released amount measured from the in vitro releasing assay using ultra performance liquid chromatography. In the releasing assay, it was estimated that $10 \mathrm{uM}(2.25 \mathrm{ug} / \mathrm{ml})$ harmine would be released from $16.98 \mu \mathrm{g}$ of harmine loaded onto $80 \mathrm{mg} \mathrm{BCP} \mathrm{(data} \mathrm{are} \mathrm{not} \mathrm{shown).} \mathrm{Before} \mathrm{transplantation,}$ hPDLCs $\left(1 \times 10^{6}\right.$ cell/ carrier) were seeded on the carrier for Groups 3, 4 and 6, and 
incubated for 2 hours. Then, the assigned combination of BCP carrier, either hPDLCs or not, and either rhBMP-2 or harmine or not was transplanted in the subcutaneous area of the dorsum in mice. The animals were sacrificed 2 or 8 weeks postsurgery.

\section{Histologic \& histomorphometric analysis}

The explanted specimens were fixed in $10 \%$ formalin, decalcified with $10 \%$ EDTA solution containing $1 \%$ paraformaldehyde, embedded in paraffin, and then sectioned at a thickness of $4 \mu \mathrm{m}$. After deparaffinization, the specimens were stained with hematoxylin-eosin and PicroSirius red, and were examined using a binocular microscope (Leica DM LB; Leica Microsystems, Wetzlar, Germany). Images of the specimens were captured and saved (cellSens Standard 1.11; Olympus Corporation, Center Valley, PA, USA). Newly formed mineralized tissue was measured using automated image analysis software (Photoshop CS6; Adobe Systems, San Jose, CA, USA). The percentage of newly formed mineralized tissue was calculated against the total augmented area. The histomorphometric analysis was performed by a single experienced investigator (H.C.L) who was blinded from the group allocation.

\section{Statistical analysis}

A software package was used for statistical analysis (SPSS ver. 21.0, IBM Corporation, Armonk, NY, USA). Shapiro-Wilk tests were performed for normal distribution of the data. Then, either ANOVA and Student`s $t$ test or Kruskal-Wallis test and Mann-Whitney U test were used. For post-hoc multiple comparison, Tukey tests for ANOVA or Dunn`s test for Kruskal-Wallis test was used. The level of statistical significance was set at $P<0.05$. 


\section{Results}

\section{Effect of harmine concentration on hPDLCs}

With 0 to $10 \mu \mathrm{M}$ of harmine, cell proliferation significantly increased with time $(P<0.05)$. There was no statistically significant difference among corresponding time points for 0 to 10 $\mu \mathrm{M}$. However, at $20 \mu \mathrm{M}$, statistically low cell proliferation was observed at 7 and 14 days, compared with the lower concentrations. Moreover, the proliferation at 7 and 14 days was significantly decreased compared to that at 3 days, and the same results were observed with $40 \mu \mathrm{M}$ (Fig. 1). Thus, $10 \mu \mathrm{M}$ harmine is suspected to be the threshold value, so we used 10 $\mu \mathrm{M}$ harmine for further assays.

\section{Osteogenic and cementogenic characteristics of hPDLC/BMP-2 and hPDLC/harmine (alizarin red S staining, real-time PCR, and Western blotting) \\ In alizarin red $\mathrm{S}$ staining, the amount of mineralization at 14 days was not significantly different among hPDLCs, hPDLC/Har and hPDLC/BMP. The mineralization in hPDLC/Har and hPDLC/BMP at 28 days significantly increased compared to 14 days, and was significantly greater than that of hPDLCs alone. There was no statistical difference between hPDLC/Har and hPDLC/BMP at 28 days (Fig. 2).}

In real-time-PCR, a highest gene expression level was observed for Runx2 in hPDLC/Har at 3, 7, 10 days. At 3 days, the level of Runx2 in hPDLC/Har was statistically higher compared to hPDLC/BMP. At 7 and 10 days, the level of Runx 2 in hPDLC/Har was statistically higher than hPDLCs alone. The level of OCN in hPDLC/Har increased more than twofold at 7 days compared to hPDLCs alone, but it was not statistically different. Also, the level of CEMP-1 became higher at 7 days than hPDLCs alone, but it did not reach statistical difference (Fig. $3 \mathrm{~A})$. 
In Western blot assay, the bands for Runx2 were thicker in hPDLC/Har than hPDLCs alone at both 7 and 14 days. At 7 days, the band for Runx 2 in hPDLC/Har was thicker compared to hPDLC/BMP. The bands for OCN and CEMP-1 in hPDLC/Har were thicker than hPDLCs alone at both 7 and 14 days (Fig. 3B).

\section{Histologic and histomorphometric analyses of ectopic transplantation}

Histologic observation at 2 weeks

In Group 1 (BCP), no mineralized tissue was observed. BCP particles were surrounded by loose connective tissue and a few inflammatory cells. In Group 2 (BCP/BMP), immature mineralized tissue and osteoid-like structure was observed on some of BCP particles with randomly oriented connective tissue. In Group 3 (BCP/hPDLC), a little mineralized tissue was seen in two specimens, but in Group 4 (BCP/hPDLC/BMP), the apposition of mineralized tissue on the particles was frequently observed in all specimens, and periodontal ligament (PDL)-like fibers were also found. In Group 5 (BCP/Har), mineralized tissue was rarely observed, and multinucleated giant cells were frequently observed in the connective tissue area. In Group 6 (BCP/hPDLC/Har), an increased amount of immature and osteoid-like

mineralized tissue was formed compared to Group 5. PDL-like fibers were observed around the immature mineralized tissue. Multinucleated cells were also observed, but fewer were present in comparison to Group 5 (Fig. 4A-L).

\section{Histologic observation at 8 weeks}

In the group 1, there was no sign of mineralization. Many blood vessels and various densities of connective tissue were observed. In Group 2, bone tissues with osteoblasts, osteocytes and reversal lines were observed with abundant fatty marrow. In Group 3, 
mineralized tissues were observed on a few BCP particles and Sharpey`s fiber-like collagen bundles were found around some mineralized tissue. Group 4 demonstrated the greatest amount of mineralized tissue. Bone tissues lining osteoblasts and containing osteocytes were commonly found around BCP particles, and cementum-like tissue with Sharpey`s fiber-like collagen insertion was also observed. Various amount of fatty marrow formation was observed in the specimens of Group 4. In Group 5, the presence of mineralized tissue was limited and dense connective tissue filled in the intergranular space. Multinucleated giant cells were observed like the finding at 2 weeks, but the amount decreased. In Group 6, two different mineralized patterns were found like in Group 4: bone tissue with randomlyoriented collagen fiber and cementum-like tissue inserted by Sharpey`s fiber-like structure. However, cementum-like tissue was more frequently observed compared to in Group 4. Varying degrees of fatty marrow formation were observed among the specimens in Group 6 (Fig. 4M-X).

Picro-Sirius red staining was performed for Groups 4 and 6 for orientation of collagen fiber near the mineralized tissue. Parallel-oriented and perpendicularly-inserted collagen fibers were observed near mineralized tissue in both groups. In Group 6, more intensive insertion of perpendicular fibers was observed, as compared to the group 4 (Fig. 5).

\section{Histomorphometry}

At 2 weeks, the amount of mineralized tissue was the greatest in Group 4, followed by Groups 6, 2, 5, 3 and 1. Compared to Groups 1, 4 and 6 demonstrated statistically greater mineralization $(P<0.05)$. Group 4 showed statistically greater mineralization than Group 3. At 8 weeks, the greatest mineralization was observed in Group 4, and mineralization of Group 2 and 6 were comparable. Groups 2, 4 and 6 had statistically greater mineralization than group 1. Group 2 and 6 showed approximately ten-fold more mineralization than Groups 3 and 5, 
but statistical significance was not found. Group 4 had statistically greater mineralization compared to Group 3 (Fig. 6, Table 1).

\section{Discussion}

The present study investigated whether harmine is a candidate for tissue regeneration using PDL cells, focused on periodontal regeneration. It was previously demonstrated that harmine has bone anabolic effect (Yonezawa et al., 2011a, Yonezawa et al., 2011b), but it has yet to reveal the effect of harmine on periodontal regeneration. Harmine treatment in vitro significantly increased mineralization nodule formation and Runx2 expression. An increase of CEMP-1 was shown, but it was not statistically significant. In the transplantation model of rats, hPDLCs with harmine increased not only bone-like but also cementum-like mineralization. Specifically, cementum-like hard tissue embedded Sharpey`s fiber-like collagen fibers was more frequently observed when PDL cells was supplemented by harmine than by rhBMP-2.

The effective concentration of harmine for hPDLCs has yet to be determined because the present study is the first to investigate the effect of harmine on hPDLCs. In MTT assay, cell toxicity was not found up to $10 \mu \mathrm{M}$ of harmine, but cell proliferation was significantly diminished at $20 \mu \mathrm{M}$. In previous studies, the effect of harmine concentration was also investigated for bone-anabolic purposes. Yonezawa et al. demonstrated that harmine decreased TRAP activity dose dependently up to $10 \mu \mathrm{M}$ for RAW264.7 cells, and $3 \mu \mathrm{M}$ almost completely inhibited multinucleated osteoclast formation (Yonezawa et al., 2011a). In the following study, a dose-dependent increase in the intensity of ALP, alizarin S and von 
Kossa staining was observed for up to $10 \mu \mathrm{M}$ of harmine in MC3T3-E1 cells, and a similar trend in ALP activity was observed in C3H10T1/2 cells (Yonezawa et al., 2011b). Thus, we determined that $10 \mu \mathrm{M}$ is a threshold for periodontal regeneration. However, it should be noted that smaller concentration of harmine was not tested for mineralization with PDLCs.

One important finding from the previous study was that ALP activity was enhanced by harmine treatment in both mesenchymal cells $(\mathrm{C} 3 \mathrm{H} 10 \mathrm{~T} 1 / 2)$ and osteoblast precursor cells (MC3T3-E1) (Yonezawa et al., 2011b). These results demonstrate that harmine not only increases the function of osteoblastic lineage cells, but also differentiates mesenchymal cells into mineralized tissue forming cells, which suggests that harmine is a potential osteoinductive agent. In the present study, harmine was compared with rhBMP-2. In mineralized nodule formation shown by Alizarin S staining, there was no statistical difference between harmine and rhBMP-2. In real-time PCR, only Runx2 gene in hPDLCs/Har was statistically greater than other groups, but the increased expression of Runx2 from 3 days indicated that osteoblastic cascade had been already initiated because Runx2 is major transcription factor for osteoblastic differentiation (Prince et al., 2001). Such may be also related with an insignificantly increased level of OCN at 7days in hPDLC/Har (real-time PCR) and thicker bands of ALP, OCN in hPDLC/Har than in hPDLC only (Western blotting). In ectopic transplantation model, Group $6(\mathrm{BCP} / \mathrm{hPDLC} / \mathrm{Har})$ enhanced mineralized tissue formation, and the amount of mineralization was statistically comparable to Group 2 (BCP/BMP) and $4(\mathrm{BCP} / \mathrm{hPDLC} / \mathrm{BMP})$. Considering the above findings, harmine could be regarded as an effective stimulant for increasing mineralization from the target cells.

One may argue that the capacity of harmine as a direct inducer for mineralization is insufficient, because there was little effect on mineralized tissue formation when it was used 
alone (Group 5). However, it was hard to say that the mineralization without target cells is surely advantageous. If some effects, e.g. mineralization is generated by a certain biomaterial in the absence of the target cells, the consequence may be unwanted tissue formation in the unwanted location. Therefore, in other way around, the stimulating action of harmine in the presence of the target cells may be regarded to be more desirable although it leaves room for further discussion on which is a better mode for the regeneration in terms of the presence or absence of target cells.

In Groups 3, 4 and 6, cementum-like mineralized tissue with Sharpey`s fiber-like collagen bundle was observed, which may be related to the finding that there was an increase of CEMP-1 in real-time PCR and Western blot assays in both rhBMP-2- and harmine-treated groups. CEMP-1 is specifically expressed by cementoblasts and PDL cells (Alvarez et al., 2007, Arzate et al., 2002). Overexpression of CEMP-1 improves cementoblast differentiation during the differentiation of periodontal ligament cells, but reduced osteoblast differentiation (Komaki et al., 2012). Notably enough, when hPDLCs were treated with harmine (Group 6), the development of such cementum-resembling structures was more frequently found compared to hPDLCs treated with rhBMP-2; Sharpey`s fiber-like collagen bundles were more intensively observed under Picro-Sirius red staining in Group 6. As described earlier, harmine significantly activated Runx2 compared to rhBMP-2, which was in line with the previous study (Yonezawa et al., 2011b). Even though Runx2 is known as a specific regulator of osteoblast differentiation, it was also observed that immunoreactivity of Runx2 was detected in acellular/cellular cementum formation (Hirata et al., 2009). Thus, it can be conjectured that the effect of harmine on hPDLCs is not oriented only in bone formation, rather dual activation in both cementum and bone formation compared to rhBMP-2. 
As in the rhBMP-2-treated groups, fatty marrow formation was observed in Group 6. It is well-known that BMP-2 stimulate adipogenesis as well as osteogenesis (Zara et al., 2011). It has been demonstrated that harmine induces BMPs expression and activates BMP pathway (Yonezawa et al., 2011b), which may indicate that harmine could induce fatty marrow formation like BMP-2.

Multinucleated giant cells were frequently observed in Groups 5 and 6 at 2 weeks in vivo compared to other groups. Even though MTT assay demonstrated that $10 \mu \mathrm{M}$ is a threshold concentration for hPDLCs in vitro, in vivo application may result in somewhat different consequences because various cells interplay in an in vivo environment. Meanwhile, Group 6 showed fewer multinucleated cells compared to Group 5, which was in accordance with results from previous studies reporting the immunosuppressive effect of stem cells (Jung et al., 2014, Le Blanc et al., 2003, Wada et al., 2009). Moreover, lower concentrations of harmine $(<10 \mu \mathrm{M})$ were not tested for mineralization in the present study, and the comparison with $1 \mu \mathrm{g}$ of rhBMP-2 might be questioned even though it is the known positive control from our previous study (Jung et al., 2014). Considering the above, further investigation about different concentrations should be performed for optimization.

Some concerns can be logically raised about the application of harmine because it has several other pharmacologic actions. It has been suggested that the bone anabolic effects of harmine are not affected by the presence of antagonists or agonists to receptors for exerting other activities (Yonezawa et al., 2011b). Such a finding may indicate that harmine delivery could provoke several events simultaneously and careful evaluation using preclinical models is further required. 
PDLC is known to consist of several phenotypes, such as cementoblast-like and/or osteoblast-like properties (Marchesan et al., 2011). In the present study, harmine enhanced mineralization with hPDLCs in vitro and in vivo, and the mineralized tissue included both bone and cementum-like tissues, which is attributable to possibly Runx 2 activation. The present study was the fisrt step for extracting potential candidate of periodontal regeneration, and should be further supported by preclinical studies with experimentally induced periodontal defect.

\section{Acknowledgement}

This paper was supported by Fund of Biomedical Research Institute, Chonbuk National University Hospital. 


\section{References}

Allen JRF and Holmstedt BR (1980). The simple $\beta$-carboline alkaloids. Phytochemistry 19: $1573-1582$.

Alvarez B, Gomez N, Jose Garrido J, Yerle M, Revilla C, Chamorro S, Alonso F, Dominguez $\mathrm{J}$ and Ezquerra A (2007). Molecular cloning characterization and expression of porcine immunoreceptor SIRPalpha. Dev Comp Immunol 31: 307-18.

Arzate H, Jimenez-Garcia LF, Alvarez-Perez MA, Landa A, Bar-Kana I and Pitaru S (2002). Immunolocalization of a human cementoblastoma-conditioned medium-derived protein. $J$ Dent Res 81: 541-6.

Berrougui H, Martin-Cordero C, Khalil A, Hmamouchi M, Ettaib A, Marhuenda E and Herrera MD (2006). Vasorelaxant effects of harmine and harmaline extracted from Peganum harmala L. seeds in isolated rat aorta. Pharmacol Res 54: 150-7.

Cao R, Peng W, Wang Z and Xu A (2007). beta-Carboline alkaloids: biochemical and pharmacological functions. Curr Med Chem 14: 479-500.

Cochran DL, Oh TJ, Mills MP, Clem DS, McClain PK, Schallhorn RA, McGuire MK, Scheyer ET, Giannobile WV, Reddy MS, Abou-Arraj RV, Vassilopoulos PJ, Genco RJ, Geurs NC and Takemura A (2016). A Randomized Clinical Trial Evaluating rh-FGF-2/beta-TCP in Periodontal Defects. J Dent Res 95: 523-30.

Cunha-Cruz J, Hujoel PP and Kressin NR (2007). Oral health-related quality of life of periodontal patients. J Periodontal Res 42: 169-76.

Dentino A, Lee S, Mailhot J and Hefti AF (2013). Principles of periodontology. Periodontol 2000 61: 16-53.

Hirata A, Sugahara $\mathrm{T}$ and Nakamura H (2009). Localization of runx2, osterix, and osteopontin in tooth root formation in rat molars. J Histochem Cytochem 57: 397-403.

Jung IH, Kwon BS, Kim SH, Shim HE, Jun CM and Yun JH (2013). Optimal medium 
formulation for the long-term expansion and maintenance of human periodontal ligament stem cells. J Periodontol 84: 1434-44.

Jung IH, Lee SH, Jun CM, Oh N and Yun JH (2014). Characterization of the enhanced bone regenerative capacity of human periodontal ligament stem cells engineered to express the gene encoding bone morphogenetic protein 2. Tissue Eng Part A 20: 2189-99.

Kassebaum NJ, Bernabe E, Dahiya M, Bhandari B, Murray CJ and Marcenes W (2014). Global burden of severe periodontitis in 1990-2010: a systematic review and meta-regression. J Dent Res 93: 1045-53.

Kim H, Sablin SO and Ramsay RR (1997). Inhibition of monoamine oxidase A by betacarboline derivatives. Arch Biochem Biophys 337: 137-42.

Kim K, Yi T and Yun JH (2016). Maintained Stemness of Human Periodontal Ligament Stem Cells Isolated After Prolonged Storage of Extracted Teeth. J Periodontol 87: e148-58.

Komaki M, Iwasaki K, Arzate H, Narayanan AS, Izumi Y and Morita I (2012). Cementum protein 1 (CEMP1) induces a cementoblastic phenotype and reduces osteoblastic differentiation in periodontal ligament cells. J Cell Physiol 227: 649-57.

Le Blanc K, Tammik L, Sundberg B, Haynesworth SE and Ringden O (2003). Mesenchymal stem cells inhibit and stimulate mixed lymphocyte cultures and mitogenic responses independently of the major histocompatibility complex. Scand J Immunol 57: 11-20.

Lee $\mathrm{J}$ and Wikesjo UM (2014). Growth/differentiation factor-5: pre-clinical and clinical evaluations of periodontal regeneration and alveolar augmentation--review. $J$ Clin Periodontol 41: 797-805.

Marchesan JT, Scanlon CS, Soehren S, Matsuo M and Kapila YL (2011). Implications of cultured periodontal ligament cells for the clinical and experimental setting: a review. Arch Oral Biol 56: 933-43.

Miron RJ, Sculean A, Cochran DL, Froum S, Zucchelli G, Nemcovsky C, Donos N, 
Lyngstadaas SP, Deschner J, Dard M, Stavropoulos A, Zhang Y, Trombelli L, Kasaj A, Shirakata Y, Cortellini P, Tonetti M, Rasperini G, Jepsen S and Bosshardt DD (2016). Twenty years of enamel matrix derivative: the past, the present and the future. J Clin Periodontol 43: $668-83$.

Moura DJ, Richter MF, Boeira JM, Pegas Henriques JA and Saffi J (2007). Antioxidant properties of beta-carboline alkaloids are related to their antimutagenic and antigenotoxic activities. Mutagenesis 22: 293-302.

Nevins M, Giannobile WV, McGuire MK, Kao RT, Mellonig JT, Hinrichs JE, McAllister BS, Murphy KS, McClain PK, Nevins ML, Paquette DW, Han TJ, Reddy MS, Lavin PT, Genco RJ and Lynch SE (2005). Platelet-derived growth factor stimulates bone fill and rate of attachment level gain: results of a large multicenter randomized controlled trial. $J$ Periodontol 76: $2205-15$.

Prince M, Banerjee C, Javed A, Green J, Lian JB, Stein GS, Bodine PV and Komm BS (2001). Expression and regulation of Runx2/Cbfa1 and osteoblast phenotypic markers during the growth and differentiation of human osteoblasts. J Cell Biochem 80: 424-40.

Takayama S, Murakami S, Shimabukuro Y, Kitamura M and Okada H (2001). Periodontal regeneration by FGF-2 (bFGF) in primate models. $J$ Dent Res 80: 2075-9.

Wada N, Menicanin D, Shi S, Bartold PM and Gronthos S (2009). Immunomodulatory properties of human periodontal ligament stem cells. J Cell Physiol 219: 667-76.

Yonezawa T, Hasegawa S, Asai M, Ninomiya T, Sasaki T, Cha BY, Teruya T, Ozawa H, Yagasaki K, Nagai K and Woo JT (2011a). Harmine, a beta-carboline alkaloid, inhibits osteoclast differentiation and bone resorption in vitro and in vivo. Eur J Pharmacol 650: 5118.

Yonezawa T, Lee JW, Hibino A, Asai M, Hojo H, Cha BY, Teruya T, Nagai K, Chung UI, Yagasaki K and Woo JT (2011b). Harmine promotes osteoblast differentiation through bone 
morphogenetic protein signaling. Biochem Biophys Res Commun 409: 260-5.

Zara JN, Siu RK, Zhang X, Shen J, Ngo R, Lee M, Li W, Chiang M, Chung J, Kwak J, Wu BM, Ting K and Soo C (2011). High doses of bone morphogenetic protein 2 induce structurally abnormal bone and inflammation in vivo. Tissue Eng Part A 17: 1389-99.

Zeichner-David M (2006). Regeneration of periodontal tissues: cementogenesis revisited. Periodontol 2000 41: 196-217. 


\section{Figure legends}

Figure 1. Cell proliferation for different concentrations of harmine (MTT assay).

*Significantly different compared to 3 days at the same concentration

${ }^{\dagger}$ Significantly different compared to 7 days at the same concentration

*Significantly different compared to corresponding time points for each concentration

Figure 2. Mineralized tissue formation capability of hPDLCs, hPDLCs/harmine (hPDLC/Har) and hPDLCs/rhBMP-2 (hPDLC/BMP). A) Representative images of alizarin red S staining following osteogenic differentiation. B) Quantitative analysis of mineralized nodule formation. The amount of mineralization was significantly greater in hPDLC/Har and hPDLC/BMP than hPDLCs at 28 days. No statistical difference was noted between hPDLC/Har and hPDLC/BMP.

*Statistically significant compared to 14 days

†Statistically significant compared to hPDLCs

Figure 3. The results of real-time PCR and Western blot assay for hPDLCs, hPDLCs/harmine (hPDLC/Har) and hPDLCs/rhBMP-2 (hPDLC/BMP).

A) Relative gene expression of alkaline phosphatase $(A L P)$, osteocalcin $(O C N)$, Runt-related transcription factor 2 (Runx2) and cementum protein 1 (CEMP1) in hPDLCs, hPDLC/Har and hPDLC/BMP quantified by real-time PCR. Runx2 in hPDLC/Har was statistically higher at 3 days compared to hPDLC/BMP, and was statistically higher at 7 and 10 days than hPDLCs alone. CEMP-1 in hPDLC/Har became insignificantly higher at 7 days than hPDLCs alone. B) Expression level of ALP, OCN, Runx2 and CEMP1 proteins in hPDLCs, hPDLC/Har and hPDLC/BMP by Western blot. The thickness of bands in Runx 2 stood out in hPDLC/Har at both 7 and 14 days. The bands for OCN and CEMP-1 in hPDLC/Har were 
thicker than hPDLCs alone at both 7 and 14 days.

†Statistically significant compared to hPDLCs/Har

Figure 4. Histologic observation of ectopic transplantation model. A-F) Observation at 2 weeks with magnification x 100. G-L) Observation at 8 weeks with magnification $\mathrm{x} 400$. MR) Observation at 8 weeks with magnification $x$ 100. S-X) Observation at 8 weeks with magnification x 400. Arrowhead, cementum-like tissue having Sharpey`s fiber-like collagen fiber insertion; Arrow, multinucleated giant cells; NM, newly-formed non-mineralized tissue; $\mathrm{NB}$, new bone; $\mathrm{BM}$, bone marrow; $\mathrm{BCP}$, biphasic calcium phosphate (BCP) carrier only; BCP/BMP, BCP carrier-loaded rhBMP-2; BCP/hPDLC, hPDLCs seeded on a BCP carrier; $\mathrm{BCP} / \mathrm{hPDLC} / \mathrm{BMP}$, hPDLCs seeded on a rhBMP-2-loaded $\mathrm{BCP}$ carrier; BCP/Har, BCP carrier-loaded harmine; BCP/hPDLC/Har, hPDLC-seeded harmine-loaded BCP carrier.

Figure 5. Histologic observation using Picro-Sirius red staining. A-C) BCP/hPDLC/BMP, hPDLCs seeded on a rhBMP-2-loaded biphasic calcium phosphate (BCP) carrier. D-F) BCP/hPDLC/Har, hPDLC-seeded harmine-loaded BCP carrier. A, D) original magnification $\mathrm{x}$ 100. B, C, E, F) original magnification x 400. Arrow head, cementum-like mineralized tissue with Sharpey`s-like fiber insertion; NB, new bone; BM, bone marrow.

Figure 6. Histomorphometric analysis of ectopic transplantation. BCP, biphasic calcium phosphate (BCP) carrier only; BCP/BMP, BCP carrier-loaded rhBMP-2; BCP/hPDLC, hPDLCs seeded on a BCP carrier; BCP/hPDLC/BMP, hPDLCs seeded on a rhBMP-2-loaded $\mathrm{BCP}$ carrier; BCP/Har, BCP carrier-loaded harmine; BCP/hPDLC/Har, hPDLC-seeded harmine-loaded BCP carrier. 
*Statistically significant compared to BCP

${ }^{\dagger}$ Statistically significant compared to BCP/hPDLC

HStatistically significant compared to 2 weeks between the groups receiving same treatment 


\section{Table}

Table 1. Histomorphometric analysis of ectopic transplantation (mean \pm SD)

\begin{tabular}{|c|c|c|c|c|c|c|}
\hline & $\begin{array}{l}\text { Group } 1 \\
(\mathrm{BCP})\end{array}$ & $\begin{array}{c}\text { Group } 2 \\
(\mathrm{BCP} / \mathrm{BMP})\end{array}$ & $\begin{array}{c}\text { Group } 3 \\
(\mathrm{BCP} / \mathrm{hPDLC})\end{array}$ & $\begin{array}{c}\text { Group } 4 \\
(\mathrm{BCP} / \mathrm{hPDSC} / \mathrm{BMP})\end{array}$ & $\begin{array}{l}\text { Group } 5 \\
(\mathrm{BCP} / \mathrm{Har})\end{array}$ & $\begin{array}{c}\text { Group } 6 \\
\text { (BCP/hPDLC/Har) }\end{array}$ \\
\hline 2 weeks & 0 & $0.08 \pm 0.09$ & $0.01 \pm 0.03$ & $0.16 \pm 0.06^{* \dagger}$ & $0.04 \pm 0.01$ & $0.13 \pm 0.11^{*}$ \\
\hline 8 weeks & 0 & $2.24 \pm 1.81^{\dagger}$ & $0.19 \pm 0.14^{\dagger}$ & $6.65 \pm 4.76^{* \dagger+}$ & $0.28 \pm 0.14^{\dagger}$ & $2.13 \pm 1.13^{* \dagger}$ \\
\hline \multicolumn{7}{|c|}{ *satistically different compared to the group 1} \\
\hline \multicolumn{7}{|c|}{${ }^{\dagger}$ statistically different compared to the group 3} \\
\hline
\end{tabular}

